\title{
Nanopartículas magnéticas de zinc $Y$ calcio para aplicaciones en hipertermia magnética
}

\author{
Magnetic nanoparticles of zinc and calcium for magnetic \\ hyperthermia application
}

\section{Nanopartículas magnéticas de zinco e cálcio para aplicações em hipertermia magnética}

Fecha de recepción: 25 de noviembre de 2015

Fecha de aprobación: 31 de marzo de 2016
Rosario Argentina Jasso-Terán Dora Alicia Cortés-Hernández** Héctor Javier Sánchez-Fuentes ${ }^{* * *}$ Pamela Yajaira Reyes-Rodríguez ${ }^{* * *}$ Laura Elena de León-Prado

\section{Resumen}

El cáncer es la segunda causa de muerte en el mundo. El tratamiento alternativo de hipertermia magnética consiste en elevar la temperatura de las células cancerígenas por medio de nanopartículas magnéticas. En este trabajo se presenta la síntesis y la caracterización de dos ferritas de zinc-calcio $\left(\mathrm{Zn}_{0.50} \mathrm{Ca}_{0.50} \mathrm{Fe}_{2} \mathrm{O}_{4}\right.$ y Zn $\left.\mathrm{Zn}_{0.25} \mathrm{Ca}_{0.75} \mathrm{Fe}_{2} \mathrm{O}_{4}\right)$. La síntesis de estas ferritas se llevó a cabo por el método de sol-gel, con posterior calcinación a $400{ }^{\circ} \mathrm{C}$. La ferrita $\mathrm{Zn}_{0.50} \mathrm{Ca}_{0.50} \mathrm{Fe}_{2} \mathrm{O}_{4}(\mathrm{ZCF} 050)$ presentó una magnetización de $31.31 \mathrm{emu} / \mathrm{g}$, y la ferrita $\mathrm{Zn}_{0.25} \mathrm{Ca}_{0.75} \mathrm{Fe}_{2} \mathrm{O}_{4}(\mathrm{ZCF} 075)$, de $38.30 \mathrm{emu} / \mathrm{g}$. El tamaño de partícula promedio fue de $14 \mathrm{~nm}$ para la ZCF050 y de $12 \mathrm{~nm}$ para la ZCF075. Adicionalmente, se realizaron pruebas de bioactividad in vitro mediante la inmersión de muestras en un fluido fisiológico simulado por 21 días bajo condiciones fisiológicas de $\mathrm{pH}$ y temperatura. Se encontró que solo la ZCF075 fue bioactiva. La habilidad de calentamiento de las ferritas se evaluó utilizando un equipo de inducción magnética en estado sólido. Las condiciones de trabajo fueron las siguientes: un campo magnético de $10.2 \mathrm{kA} / \mathrm{m}$ y una frecuencia de $362 \mathrm{kHz}$. La ferrita ZCF050 alcanzó una temperatura de $41.2^{\circ} \mathrm{C}$, utilizando una concentración de ferrita/agua de $20 \mathrm{mg} / 2 \mathrm{ml}$. La ferrita ZCF075 no logró alcanzar los $40^{\circ} \mathrm{C}$. Los resultados obtenidos del análisis

\footnotetext{
* M.Sc. Centro de Investigación y de Estudios Avanzados del Instituto Politécnico Nacional, CINVESTAV-Unidad Saltillo (Coahuila, México).

** Ph.D. Centro de Investigación y de Estudios Avanzados del Instituto Politécnico Nacional, CINVESTAV-Unidad Saltillo (Coahuila, México). dora.cortes@cinvestav.mx.

*** M.Sc. Centro de Investigación y de Estudios Avanzados del Instituto Politécnico Nacional, CINVESTAV-Unidad Saltillo (Coahuila, México).

**** M.Sc. Centro de Investigación y de Estudios Avanzados del Instituto Politécnico Nacional, CINVESTAV-Unidad Saltillo (Coahuila, México).

***** Centro de Investigación y de Estudios Avanzados del Instituto Politécnico Nacional, CINVESTAV-Unidad Saltillo (Coahuila, México).
} 
de la ferrita ZCF050 indicaron que es un material potencial para su uso en tratamientos de cáncer por hipertermia magnética.

Palabras clave: biomateriales, ferritas de zinc y calcio, fluido fisiológico simulado, hipertermia magnética.

\section{Abstract}

Cancer is the second cause of death in the world. Magnetic hyperthermia is an alternative treatment which consists of raising the temperature of cancer cells using magnetic nanoparticles. In this work, the synthesis and characterization of two calcium-zinc ferrites $\left(\mathrm{Zn}_{0.50} \mathrm{Ca}_{0.50} \mathrm{Fe}_{2} \mathrm{O}_{4}\right.$ y $\left.\mathrm{Zn}_{0.25} \mathrm{Ca}_{0.75} \mathrm{Fe}_{2} \mathrm{O}_{4}\right)$ are presented. These ferrites were synthesized by sol-gel method, afterwards they were calcined at $400{ }^{\circ} \mathrm{C}$. The saturation magnetization values were 31.31 and $38.30 \mathrm{emu} / \mathrm{g}$ for $\mathrm{Zn}_{0.50} \mathrm{Ca}_{0.50} \mathrm{Fe}_{2} \mathrm{O}_{4}$ (ZCF050) and $\mathrm{Zn}_{0.25} \mathrm{Ca}_{0.75} \mathrm{Fe}_{2} \mathrm{O}_{4}$ (ZCF075, respectively. The average particle size was $14 \mathrm{~nm}$ for ZCF050 and $12 \mathrm{~nm}$ for ZCF075. Additionally, in vitro bioactivity assessment was performed by immersing samples in simulated body fluid (SBF) for 21 days under physiological conditions of $\mathrm{pH}$ and temperature. Only the ZCF075 ferrite showed to be bioactive. The heating capacity of ferrites was evaluated under an appropriate magnetic field using solid state magnetic induction. The working conditions were the following: A magnetic field of $10.4 \mathrm{KA} / \mathrm{m}$ and a frequency of $362 \mathrm{KHz}$. The ZCF050 ferrite reached a temperature of $41.2{ }^{\circ} \mathrm{C}$ using a concentration of ferrite/water $20 \mathrm{mg} / 2 \mathrm{ml}$. The ZCF075 ferrite failed to reach 40 ${ }^{\circ} \mathrm{C}$. The results obtained in the ferrite ZCF050 indicated that these nanoparticles are potential materials for cancer treatment by magnetic hyperthermia therapy.

Keywords: biomaterials, magnetic hyperthermia, simulated body fluid, zinc and calcium ferrites.

\section{Resumo}

O câncer é a segunda causa de morte no mundo. $\mathrm{O}$ tratamento alternativo de hipertermia magnética consiste em elevar a temperatura das células cancerígenas por meio de nanopartículas magnéticas. Neste trabalho se apresenta a síntese e a caracterização de duas ferritas de zinco-cálcio $(\mathrm{Zn} 0.50 \mathrm{Ca} 0.50 \mathrm{Fe} 2 \mathrm{O} 4$ e Zn0.25Ca0.75Fe2O4). A síntese destas ferritas realizou-se pelo método de sol-gel, com posterior calcinação a $400{ }^{\circ} \mathrm{C}$. A ferrita $\mathrm{Zn} 0.50 \mathrm{Ca} 0.50 \mathrm{Fe} 2 \mathrm{O} 4$ (ZCF050) apresentou uma magnetização de $31.31 \mathrm{emu} / \mathrm{g}$, e a ferrita $\mathrm{Zn0.25Ca0.75Fe2O4} \mathrm{(ZCF075),} \mathrm{de} 38.30$ emu/g. O tamanho médio de partícula foi de $14 \mathrm{~nm}$ para a ZCF050 e de $12 \mathrm{~nm}$ para a ZCF075. Adicionalmente, se realizaram provas de bioatividade in vitro mediante a imersão de amostras em um fluido fisiológico simulado por 21 dias sob condições fisiológicas de pH e temperatura. Encontrou-se que só a ZCF075 foi bioativa. A habilidade de aquecimento das ferritas avaliou-se utilizando um equipamento de indução magnética de estado sólido. As condições de trabalho foram as seguintes: um campo magnético de $10.2 \mathrm{kA} / \mathrm{m}$ e uma frequência de $362 \mathrm{kHz}$. A ferrita ZCF050 alcançou uma temperatura de $41.2^{\circ} \mathrm{C}$, utilizando uma concentração de ferrita/água de $20 \mathrm{mg} / 2 \mathrm{ml}$. A ferrita ZCF075 não logrou alcançar os $40^{\circ} \mathrm{C}$. Os resultados obtidos da análise da ferrita ZCF050 indicaram que é um material potencial para uso em tratamentos de câncer por hipertermia magnética.

Palavras chave: biomateriais, ferritas de zinco e cálcio, fluido fisiológico simulado, hipertermia magnética.

Cómo citar este artículo:

[1] R. A. Jasso-Terán, D. A. Cortés-Hernández, H. J. Sánchez-Fuentes, P. Y. Reyes-Rodríguez and L. E. LeónPrado, "Nanopartículas magnéticas de zinc y calcio para aplicaciones en hipertermia magnética", Fac. Ing., vol. 25 (42), pp. 89-98, may.-ago. 2016. 


\section{INTRODUCCIÓN}

El cáncer es una de las enfermedades que tienen un alto índice de mortalidad; de él, existen más de 100 tipos diferentes. De acuerdo con la Organización Mundial de la Salud (OMS), tan solo en América se registran 3 millones de nuevos casos anualmente. Por tal motivo, se buscan tratamientos alternativos que puedan ayudar a combatir esta enfermedad. El desarrollo de nuevos materiales ha conducido a una mejora en los tratamientos existentes, ejemplo de ello es el tratamiento de la hipertermia, que, en un principio, consistía en elevar la temperatura de todo el cuerpo entre 42 y $47{ }^{\circ} \mathrm{C}$ para eliminar el cáncer; esto ocurrió a finales de 1800 [1]. En los últimos años, mediante el uso de los nuevos materiales y la nanotecnología, este tratamiento ha cambiado, y hoy se utilizan nanopartículas magnéticas que pueden ser transportadas a la cercanía de los tejidos cancerosos para realizar un tratamiento de hipertermia más localizado. El campo magnético no es absorbido por los tejidos vivos y puede ser aplicado en una región profunda del cuerpo [2]. Por ello, las nanopartículas magnéticas (NPM) han recibido mayor atención, dada su potencial aplicación biomédica [3]; cuando estas nanopartículas se someten a un campo magnético variable, se libera calor, debido, entre otros fenómenos, a la pérdida de histéresis. La cantidad de calor generado depende de la naturaleza de los materiales magnéticos. Para su aplicación en el tratamiento de tumores de hueso, este tipo de materiales deben ser no solo biocompatibles, sino también bioactivos. Se han realizado numerosos trabajos de investigación utilizando nanopartículas magnéticas con el fin de manifestar el efecto terapéutico en varios tipos de tumores, dichos experimentos se han realizado en animales o en cultivos de células cancerosas [2].

Existe una amplia gama de nanopartículas magnéticas que se están utilizando para desarrollar tratamientos médicos; se han investigado óxidos simples y complejos, en virtud de su potencial aplicación para el tratamiento de hipertermia [4]. La magnetita $\left(\mathrm{Fe}_{3} \mathrm{O}_{4}\right)$ es uno de los nanomateriales más investigados; también se encuentran las ferritas complejas, tales como las de cobalto $\left(\mathrm{CoFe}_{2} \mathrm{O}_{4}\right)$, las de manganeso $\left(\mathrm{MnFe}_{2} \mathrm{O}_{4}\right)$, las de níquel $\left(\mathrm{NiFe}_{2} \mathrm{O}_{4}\right)$, las de litio $\left(\mathrm{Li}_{0.5} \mathrm{Fe}_{2.5} \mathrm{O}_{4}\right)$, las mixtas de níquel-zinc-cobre $\left(\mathrm{Ni}_{0.65} \mathrm{Zn}_{0.35} \mathrm{Cu}_{0.1} \mathrm{Fe}_{1.9} \mathrm{O}_{4}\right)$, las de cobalto-níquel $\left(\mathrm{Co}_{\mathrm{x}} \mathrm{Ni}_{(1-\mathrm{x})} \mathrm{Fe}_{2} \mathrm{O}_{4}\right)$ y las de cobalto-zinc $\left(\mathrm{Ni}_{(\mathrm{x})} \mathrm{Zn}_{(1-\mathrm{x})} \mathrm{Fe}_{2} \mathrm{O}_{4}\right)$. También están las nanopartículas ferromagnéticas de hierro dopadas con $\mathrm{Au}$ y de óxido de hierro dopadas con $\mathrm{Zn}-\mathrm{Mn}\left(\mathrm{Zn}_{\mathrm{x}} \mathrm{Mn}_{(1-\mathrm{x})} \mathrm{Fe}_{3} \mathrm{O}_{4}\right)$ [5-9].

Estas nanopartículas pueden ser utilizadas, por ejemplo, en imágenes de contraste por resonancia magnética, para liberación de medicamento $\mathrm{y}$, por supuesto, para hipertermia magnética. El tamaño de las nanopartículas juega un papel importante para aplicaciones biomédicas, ya que, de acuerdo con la literatura, se pueden utilizar nanoparticulas con un tamaño entre 10 y $100 \mathrm{~nm}$; si se excede este tamaño, las NPM pueden causar problemas al introducirse en el cuerpo. Otro de los aspectos por considerar es la citotoxicidad del material, ya que si resulta tóxico no puede ser utilizado en el cuerpo.

En este trabajo se presenta la síntesis por sol-gel y la caracterización de dos ferritas de zinc-calcio con un tratamiento térmico realizado a $400{ }^{\circ} \mathrm{C}$, las cuales presentaron propiedades y características potenciales para su uso en el tratamiento de cáncer por hipertermia magnética.

\section{Materiales y MÉtodos}

Los reactivos químicos que se utilizaron fueron grado reactivo de la marca Sigma-Aldrich; fueron: $\mathrm{Zn}\left(\mathrm{NO}_{3}\right)_{2} \cdot 6 \mathrm{H}_{2} \mathrm{O} \quad(98 \%), \quad \mathrm{Ca}\left(\mathrm{NO}_{3}\right)_{2} \cdot 4 \mathrm{H}_{2} \mathrm{O} \quad(99 \%)$, $\mathrm{Fe}\left(\mathrm{NO}_{3}\right)_{2} \cdot 9 \mathrm{H}_{2} \mathrm{O}(99.99 \%)$ y etilenglicol $\left(\mathrm{C}_{2} \mathrm{H}_{6} \mathrm{O}_{2}\right.$, 99\%).

Cantidades estequiométricas de los reactivos químicos apropiados se mezclaron con etilenglicol $\left(\mathrm{C}_{2} \mathrm{H}_{6} \mathrm{O}_{2}\right)$ bajo una agitación magnética durante 2 horas a $40^{\circ} \mathrm{C}$, posteriormente se elevó la temperatura a $80^{\circ} \mathrm{C}$ hasta obtener un gel con una tonalidad café obscuro. El gel fue añejado durante 2 horas y luego se puso a secar, en una estufa de calentamiento, durante 72 horas, a $95{ }^{\circ} \mathrm{C}$. Finalmente, al gel precursor se le realizó un tratamiento térmico a $400{ }^{\circ} \mathrm{C}$ durante $30 \mathrm{~min}$, con el objetivo de obtener las ferritas mixtas propuestas.

Los materiales obtenidos fueron analizados por difracción de rayos X (DRX, Philips 3040), magnetometría de muestra vibrante a temperatura ambiente (MMV, Princeton Measurements Corporation, MicroMag ${ }^{\mathrm{TM}}$ 2900), microscopía electrónica de barrido (MEB, JSM 6300, Jeol), espectroscopía por dispersión de energía (EDS) y microscopía electrónica de transmisión (MET, FEI Titan 80-300). 
Otra de las pruebas que se les realizaron a las muestras fue la de bioactividad in vitro, que consiste en sumergir las muestras en $150 \mathrm{~mL}$ de un fluido fisiológico simulado (FFS) durante diferentes periodos de inmersión (7, 14 y 21 días). Lo anterior bajo condiciones fisiológicas de $\mathrm{pH}$ y temperatura. La concentración iónica del FFS utilizado y del plasma sanguíneo se encuentra en la Tabla 1.
Uno de los objetivos primordiales de esta investigación es que las nanopartículas sean capaces de elevar la temperatura por medio de un campo magnético oscilatorio. Para evaluar esta capacidad de calentamiento se utilizó un equipo de inducción magnética en estado sólido (Ambrell, EasyHeat, 0224), operado a $10.2 \mathrm{kA} / \mathrm{m}$ y una frecuencia de 362 $\mathrm{kHz}$, el líquido de enfriamiento utilizado es agua desionizada. Las pruebas se realizaron durante un periodo de $15 \mathrm{~min}$ a $200 \mathrm{~A}$. Se utilizaron diferentes concentraciones ferrita/agua desionizada.

\section{TABLA 1}

CONCENTRACIÓN IÓNICA DEL FLUIDO FISIOLÓGICO SIMULADO Y DEL PLASMA SANGUÍNEO [10]

\begin{tabular}{|l|l|l|l|l|l|l|l|l|}
\hline Concentración (mM) & $\mathbf{N a}^{+}$ & $\mathbf{K}^{+}$ & $\mathbf{M g}^{2+}$ & $\mathbf{C a}^{2+}$ & $\mathbf{C l}^{-}$ & $\mathbf{H C O}_{3}^{-}$ & $\mathbf{H P O}_{4}^{2-}$ & $\mathbf{S O}_{4}^{2-}$ \\
\hline FFS & 142.0 & 5.0 & 1.5 & 2.5 & 147.8 & 4.2 & 1.0 & 0.5 \\
\hline Plasma sanguíneo & 142.0 & 5.0 & 1.5 & 2.5 & 103.0 & 2.7 & 1.0 & 0.5 \\
\hline
\end{tabular}

\section{RESULTADOS Y DISCUSIÓN}

En la Fig. 1 se presentan los resultados obtenidos de los patrones de difracción de las ferritas $\mathrm{Zn}_{0.50} \mathrm{Ca}_{0.50} \mathrm{Fe}_{2} \mathrm{O}_{4}$ (ID: ZCF050-400) y $\mathrm{Zn}_{0.25} \mathrm{Ca}_{0.75} \mathrm{Fe}_{2} \mathrm{O}_{4}$ (ID: ZCF075400); esta figura también contiene los patrones de difracción de las ferritas $\mathrm{ZnFe}_{2} \mathrm{O}_{4}$ (JCPDS 22-1012) y $\mathrm{CaFe}_{2} \mathrm{O}_{4}$ (JCPDS32-0168). Las líneas punteadas en color negro corresponden a la ferrita de zinc, y las líneas punteadas en color gris, a la ferrita de calcio. En el patrón de difracción de la ferrita identificada como ZCF050-400 se puede observar un ligero desplazamiento hacia la derecha, en comparación con las ferritas puras, esto es un indicativo de que se está formando una ferrita mixta.

La ferrita ZCF075-400 presenta un comportamiento similar al de la ferrita anterior, y presenta ligeros desplazamientos en los ángulos de reflexión, en comparación con las cartas de difracción de las ferritas puras $\left[\mathrm{ZnFe}_{2} \mathrm{O}_{4}\right.$ (JCPDS 22-1012) y $\mathrm{CaFe}_{2} \mathrm{O}_{4}$ (JCPDS32-0168)]. Observar estos desplazamientos en los patrones de DRX es importante, debido a que nos indica que se está llevando a cabo la formación de una sola fase, una ferrita mixta.

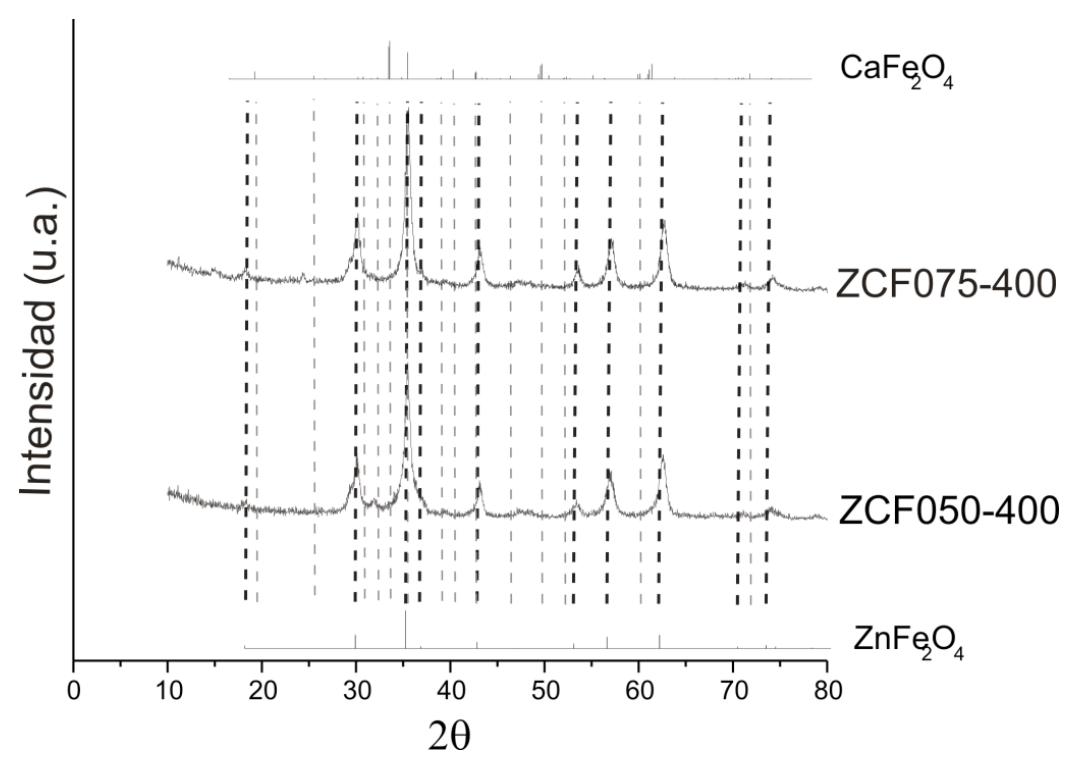

Fig. 1. Patrones de difracción de las ferritas ZCF050-400 y ZCF075-400. 
Una de las propiedades que deben tener las nanopartículas es que sean magnéticas, debido a que su objetivo es que las ferritas puedan calentar por medio de la inducción de un campo magnético. Las propiedades magnéticas de estas ferritas fueron las siguientes: la ferrita ZCF50-400 presentó una magnetización de saturación (Ms) de 31.31 $\mathrm{emu} / \mathrm{g}$, una remanencia $(\mathrm{Mr})$ de $0.45 \mathrm{emu} / \mathrm{g}$ y una coercitividad $(\mathrm{Hc})$ de 4.85 Oe. Por otro lado, la ferrita ZCF075-400 presentó una Ms de $38.30 \mathrm{emu} / \mathrm{g}$, una $\mathrm{Mr}$ de $1.48 \mathrm{em} / \mathrm{g}$ y una $\mathrm{Hc}$ de 14.02 Oe. Esta ferrita presentó propiedades magnéticas ligeramente mayores que la ZCF50-400, siendo la única diferencia la concentración de Ca y Zn. Esto puede deberse a que la ferrita de zinc presenta una estructura tipo espinela normal que, cuando se une a otro catión para formar una ferrita mixta, puede cambiar, dando lugar a una ferrita parcialmente invertida. El zinc tiene un gran efecto sobre las propiedades magnéticas, ya que la sustitución con un catión no metálico, como el zinc, que tiene preferencia por ocupar los sitios tetraédricos, resulta en una reducción en el intercambio entre los cationes de las posiciones tetraédricas y octaédricas; esto varía dependiendo del grado de sustitución del zinc; por lo tanto, a mayor contenido de zinc las propiedades magnéticas decrecen [11].
En la Fig. 2 se presentan las imágenes de MET de las ferritas ZCF050-400 y ZCF075-400, con sus respectivos patrones de difracción. En la Fig. 2(a) se puede observar que las NPM presentan una morfología irregular, y que se encuentran aglomeradas debido a su alta magnetización y a que el material es policristalino. La determinación del tamaño de partícula se obtuvo mediante el programa Gatan Digital Micrograph, resultando un tamaño de partícula promedio de $14 \mathrm{~nm}$.

En la Figura 2(b) se presentan las imágenes de MET de la ferrita ZCF075-400; se puede observar que la ferrita presenta una morfología mayormente esférica, e igual que en la figura 2(a), el patrón de difracción obtenido indica que el material es policristalino y el tamaño de partícula promedio fue de $12 \mathrm{~nm}$; este se determinó, al igual que en el caso anterior, mediante el programa Gatan Digital Micrograph. De acuerdo con la literatura [2], las nanopartículas que tienen un tamaño de partícula en un rango de 10-100 $\mathrm{nm}$ son óptimas para ser inyectadas vía intravenosa, y pueden prolongar su tiempo de circulación en la sangre; esto indica que los materiales obtenidos podrían ser utilizados en aplicaciones biomédicas.

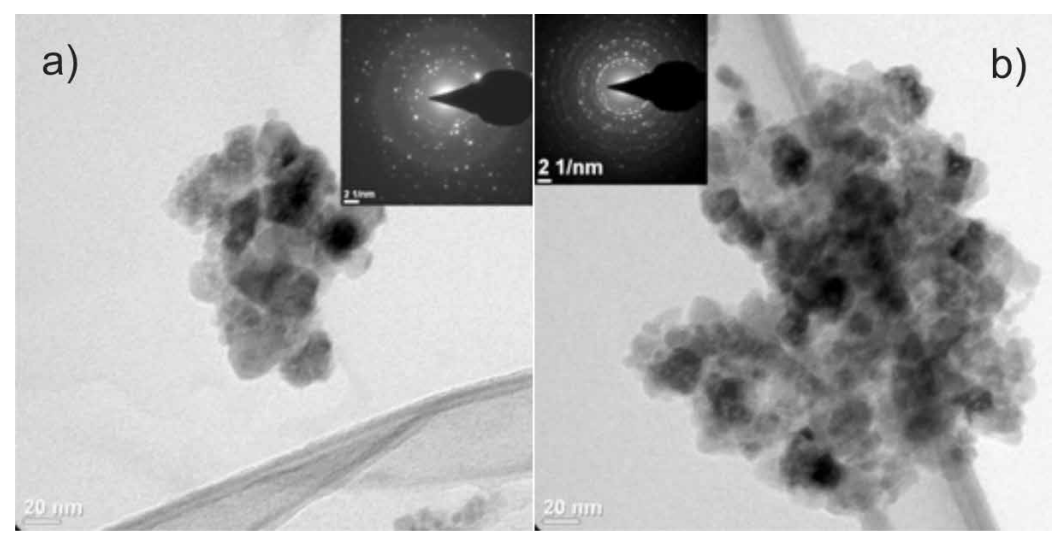

Fig. 2. Imágenes de MET (a) ferrita ZCF050-400 y (b) ferrita ZFC075-400.

El ensayo de bioactividad in vitro se realizó con la finalidad de predecir lo que ocurriría si las nanopartículas permanecieran en el cuerpo humano después del tratamiento. En caso de ser bioactivas, su potencial aplicación sería en el tratamiento de cáncer óseo mediante hipertermia.

En la Fig. 3 se presentan las imágenes de MEB de la muestra ZCF050-400 en forma de pastilla después de 21 días de inmersión en FFS (3a, b) y en polvo (3c, d). Este análisis se realizó también en polvo, debido a que se espera introducir este material en esta forma. En la imagen de la Fig. 3a no se observa la formación de algún compuesto, sin embargo, el correspondiente espectro EDS (Fig. 3b) indica la presencia de calcio y fósforo. La relación de $\mathrm{Ca} / \mathrm{P}$ que se obtuvo fue de 2.38 , considerablemente más alta que la de la hidroxiapatita, seguramente debido al contenido de $\mathrm{Ca}$ en la ferrita. Se observó este mismo comportamiento en la muestra en polvo de esta ferrita (Fig. 3c), donde la relación $\mathrm{Ca} / \mathrm{P}$ 
fue de 5.73, lo que nuevamente indica que mediante EDS se está detectando también el calcio contenido en la ferrita. Con base en la literatura [12], las capas que se forman en los compuestos bioactivos existentes en contacto con fluidos fisiológicos o reales deben presentar una relación $\mathrm{Ca} / \mathrm{P}$ cercana al 1.67 , ya que este valor está reportado para la hidroxiapatita ideal.

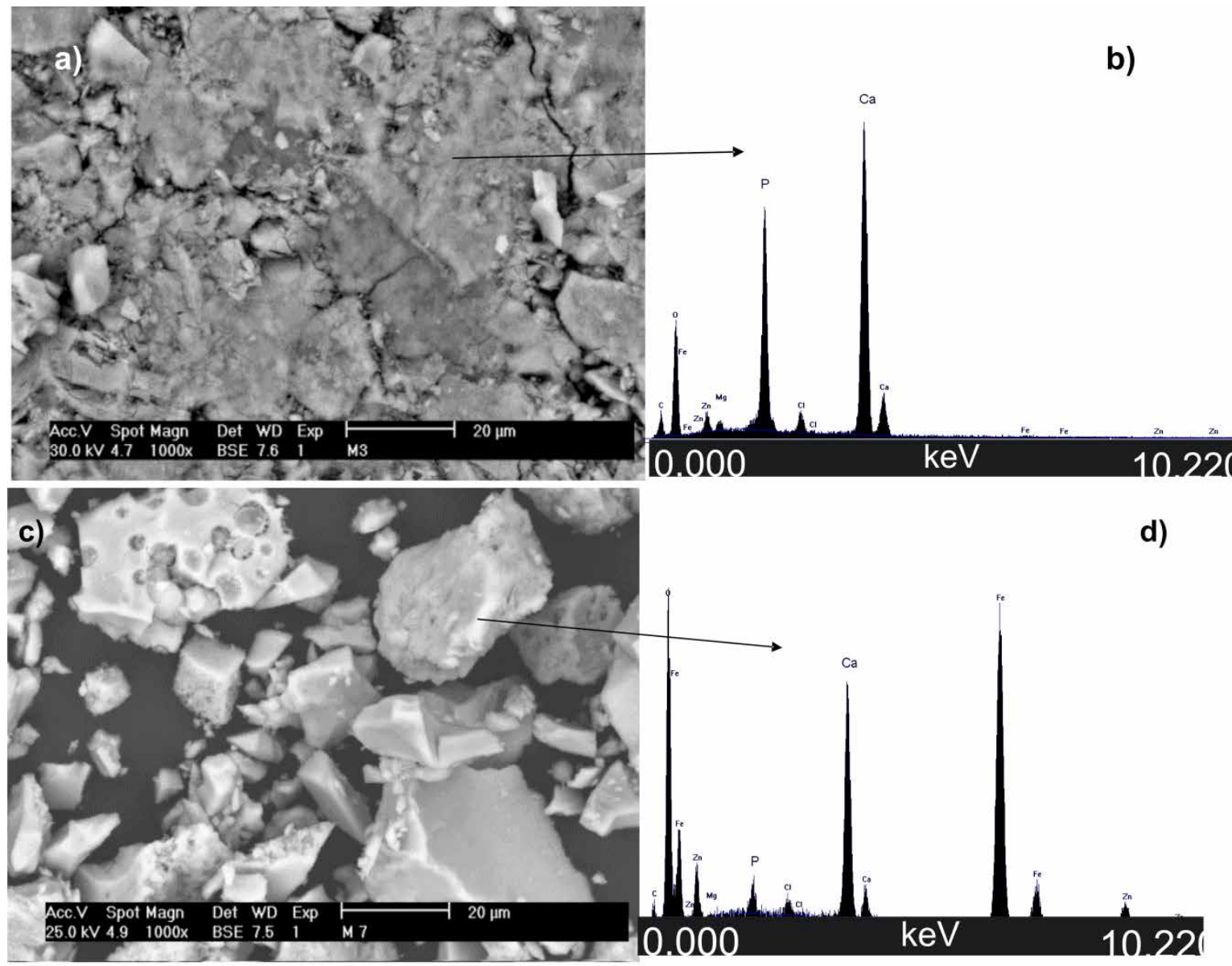

Fig. 3. Imágenes de MEB y correspondientes espectros EDS de ZCF050-400 después de 21 días de inmersión en FFS conformada en forma de pastilla $(a, b)$ y en polvo $(c, d)$.

En la Fig. 4 se presenta la imagen de MEB de la muestra ZCF075-400 después de 21 días de inmersión en forma de pastilla (Fig. 4a, b) y en polvo (4c, d). En la Fig. 4a se puede observar una capa constituida por esferas de distintos tamaños que, de acuerdo con el espectro de EDS (Fig. 4b), presenta una relación $\mathrm{Ca} / \mathrm{P}$ de 1.68 , que se encuentra dentro del rango del de las apatitas óseas; también indica que el contenido de calcio en la muestra promueve la bioactividad y que con bajas adiciones de zinc en la muestra, la bioactividad no se ve afectada.
En el caso de la imagen de MEB de la Figura 4c es posible observar estas esferas dispersas entre los aglomerados de las nanopartículas, pese a que esta muestra se encuentra en forma de polvo. La relación $\mathrm{Ca} / \mathrm{P}$ que proporciona el espectro de EDS correspondiente a la Fig. $4 \mathrm{~d}$ es de 1.82, valor que es un poco más elevado que el obtenido por la muestra en pastilla; esto se debe a que en este análisis es más fácil detectar también el calcio presente en la muestra. Con estos resultados podemos decir que esta ferrita resulta ser bioactiva, a diferencia de la anterior, indicando que el contenido de $\mathrm{Ca}-\mathrm{Zn}$ es el adecuado para la formación de apatita. 


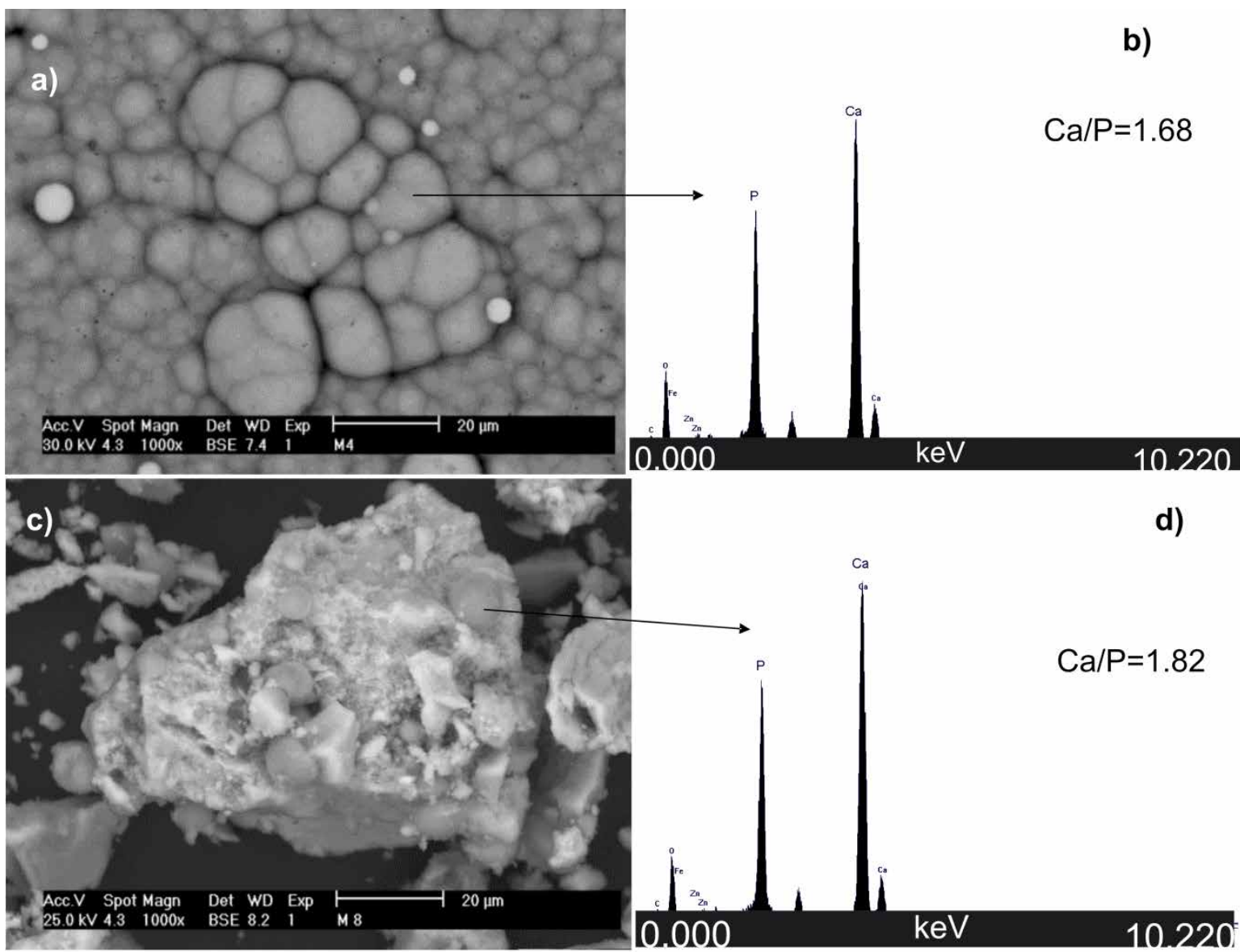

Fig. 4. Imágenes de MEB y correspondientes espectros EDS de ZCF075-400 después de 21 días de inmersión en FFS conformada en forma de pastilla $(a, b)$ y en polvo $(c, d)$.

A continuación se presentan los resultados obtenidos de las pruebas de hipertermia magnética; este análisis, como ya se mencionó, resulta ser de gran importancia, debido a que se espera que las nanopartículas tenga la capacidad de generar calentamiento cuando se sometan a un campo magnético oscilatorio. En la Fig. 5 y la Tabla 2 se presentan los resultados obtenidos de las pruebas de hipertermia magnética de la ferrita ZCF050-400. Para este análisis se utilizaron cinco suspensiones: $12,14,16,18$ y $20 \mathrm{mg}$ en $2 \mathrm{ml}$ de agua desionizada, con un tiempo de duración de prueba de 15 $\mathrm{min}$. Es importante mencionar que las concentraciones utilizadas en este análisis se encuentran dentro del rango permitido para aplicaciones in vivo, esto de acuerdo con la literatura [13].
En la Fig. 5 es posible observar que las muestras fueron incrementando su temperatura de manera gradual, conforme transcurría el tiempo de la prueba. En este caso, la única muestra que alcanzó una temperatura dentro del rango del requerido para hipertermia magnética $\left(41-46^{\circ} \mathrm{C}\right)$ fue la de $20 \mathrm{mg}$ en $2 \mathrm{ml}$, con una temperatura de $41.2^{\circ} \mathrm{C}$. El resto de las muestras presentaron un calentamiento ascendente, pero alcanzaron temperaturas de calentamiento en un rango de 36 a $40{ }^{\circ} \mathrm{C}$. Esta muestra resultó no ser bioactiva, y aunque es deseable que el material sea potencialmente bioactivo, no es una limitante para que pueda ser utilizado en el tratamiento de cáncer [11]. 


\section{TABLA 2}

DATOS DE LOS RESULTADOS DE INDUCCIÓN MAGNÉTICA DE LA FERRITA ZCF050-400

\begin{tabular}{|l|l|}
\hline Muestra & $\begin{array}{l}\text { Temperatura máxima } \\
\text { alcanzada }\left({ }^{\circ} \mathbf{C}\right)\end{array}$ \\
\hline $12 \mathrm{mg} / 2 \mathrm{ml}$ & 36.6 \\
\hline $14 \mathrm{mg} / 2 \mathrm{ml}$ & 36.9 \\
\hline $16 \mathrm{mg} / 2 \mathrm{ml}$ & 38.6 \\
\hline $18 \mathrm{mg} / 2 \mathrm{ml}$ & 40.4 \\
\hline $20 \mathrm{mg} / 2 \mathrm{ml}$ & 41.2 \\
\hline
\end{tabular}

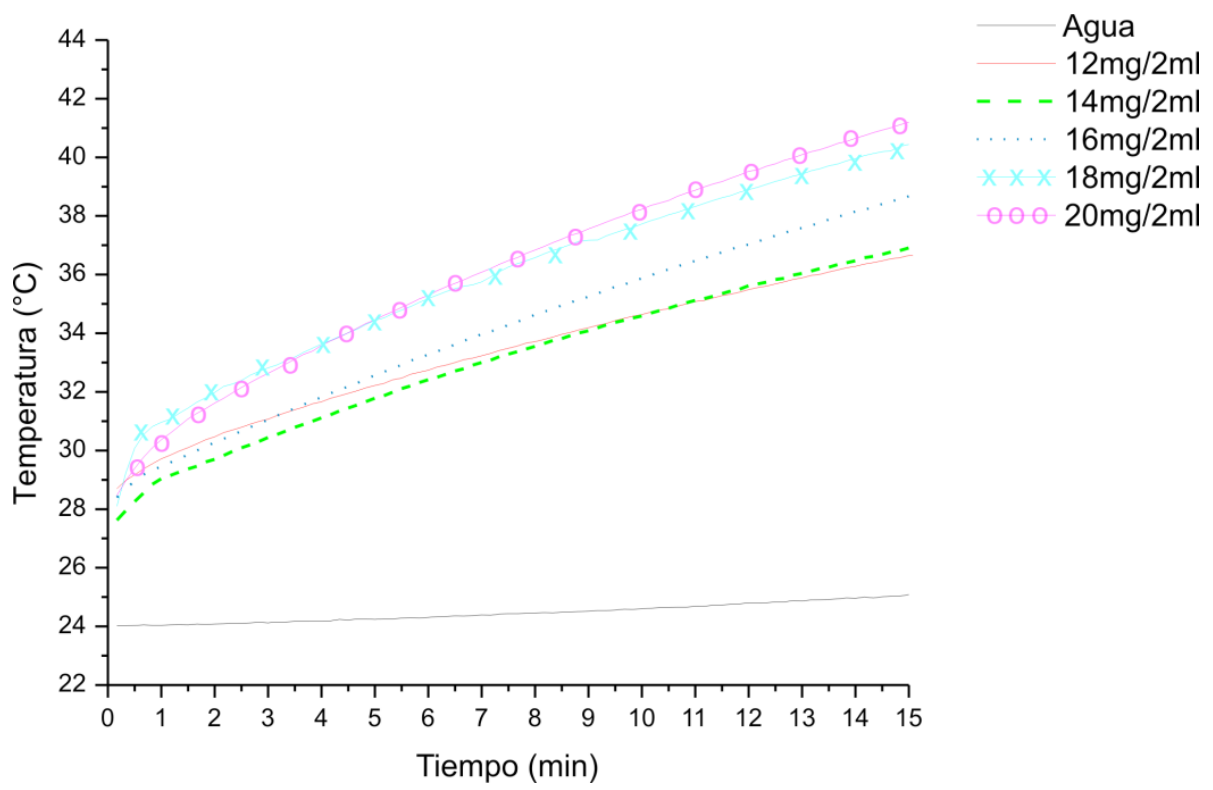

Fig. 5. Resultados de hipertermia magnética de la ferrita ZCF050-400.

En la Fig. 6 y la Tabla 3 se presentan los resultados de las pruebas de inducción magnética de la ferrita ZCF075-400. Las condiciones a las que se realizaron las pruebas fueron iguales que en el caso anterior, utilizando cinco muestras que van de los 12 a los $20 \mathrm{mg}$ de ferrita en $2 \mathrm{ml}$ de agua desionizada y con un tiempo de prueba de 15 min. Estas muestras incrementaron su temperatura gradualmente, pero, infortunadamente, no lograron alcanzar los $41^{\circ} \mathrm{C}$ para entrar dentro del rango de trabajo de la hipertermia. Las temperaturas máximas alcanzadas por estas suspensiones oscilaron entre los 34.3 y los $38^{\circ} \mathrm{C}$; por lo tanto, esta ferrita no puede ser utilizada para tratamientos de hipertermia, aunque sí podría utilizarse para otras aplicaciones, como la liberación de fármacos, en virtud de que esta ferrita presenta apropiadas propiedades magnéticas y de bioactividad. Es importante mencionar que, para su aplicación como sistema de liberación de medicamentos, se requiere la modificación o funcionalización de las nanopartículas. 


\section{TABLA 3}

DATOS DE LOS RESULTADOS DE INDUCCIÓN MAGNÉTICA DE LA FERRITA ZCF075-400.

\begin{tabular}{|l|l|}
\hline Muestra & $\begin{array}{c}\text { Temperatura máxima } \\
\text { alcanzada }\left({ }^{\circ} \mathbf{C}\right)\end{array}$ \\
\hline $12 \mathrm{mg} / 2 \mathrm{ml}$ & 34.3 \\
\hline $14 \mathrm{mg} / 2 \mathrm{ml}$ & 35.5 \\
\hline $16 \mathrm{mg} / 2 \mathrm{ml}$ & 34.6 \\
\hline $18 \mathrm{mg} / 2 \mathrm{ml}$ & 36.1 \\
\hline $20 \mathrm{mg} / 2 \mathrm{ml}$ & 38 \\
\hline
\end{tabular}

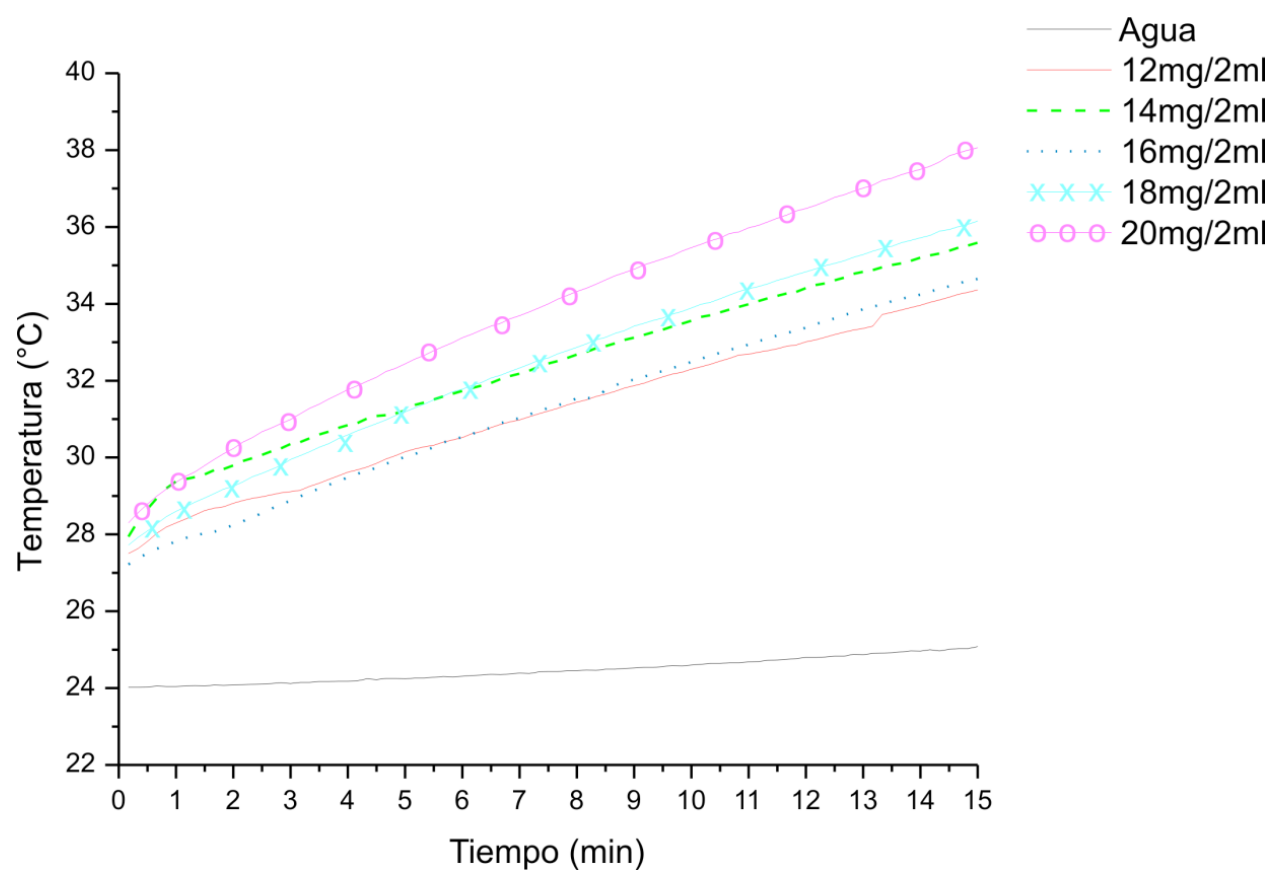

Fig. 6. Resultados de hipertermia magnética de la ferrita ZCF075-400.

\section{Conclusiones}

Fue posible sintetizar dos ferritas mixtas de zinc y calcio por medio del método de sol-gel seguido de tratamiento térmico a baja temperatura $\left(400^{\circ} \mathrm{C}\right)$. Cada uno de estos materiales presentó una fase única, un tamaño de partícula entre 12 y $14 \mathrm{~nm}$ y propiedades magnéticas similares. La ferrita ZCF050-400 (mismo contenido de $\mathrm{Ca}$ y $\mathrm{Zn}$ ) presentó mayor habilidad de calentamiento $\left(41^{\circ} \mathrm{C}\right) \mathrm{y}$, por lo tanto, un uso potencial en el tratamiento cáncer mediante hipertermia magnética, cuando se utilizaron suspensiones con una concentración de $20 \mathrm{mg}$ de ferrita en $2 \mathrm{ml}$ de agua desionizada. Por otro lado, la ferrita ZCF075-400 resultó ser altamente bioactiva, debido a su mayor contenido de calcio.

\section{REFERENCIAS}

[1] P. Cherukuri, E. S. Glazer and S. A. Curley, "Targeted hyperthermia using metal nanoparticles," Advanced drug delivery reviews, vol. 62 (3), pp. 339-345, Mar. 2010. DOI: http:// dx.doi.org/10.1016/j.addr.2009.11.006.

[2] A. K. Gupta and M. Gupta, "Synthesis and surface engineering of iron oxide nanoparticles for biomedical applications", Biomaterials, vol. 26 (18), pp. 3995-4021, Jun. 2005. DOI: http:// dx.doi.org/10.1016/j.biomaterials.2004.10.012.

[3] A. Tampieri, T. D'Alessandro, M. Sandri et al., "Intrinsic magnetism and hyperthermia in bioactive Fe-doped hydroxyapatite", Acta biomaterialia, vol. 8 (2), pp. 843-851, Feb. 2012. DOI: http://dx.doi.org/10.1016/j. actbio.2011.09.032. 
[4] C. S. Kumar and F. Mohammad, "Magnetic nanomaterials for hyperthermia-based therapy and controlled drug delivery", Advanced drug delivery reviews, vol. 63 (9), pp. 789-808, Aug. 2011. DOI: http://dx.doi.org/10.1016/j. addr.2011.03.008.

[5] M. Gharagozlou, "Synthesis, characterization and influence of calcination temperature on magnetic properties of nanocrystalline spinel Co-ferrite prepared by polymeric precursor method", Journal of Alloys and Compounds, vol. 486 (1-2), pp. 660-665, Nov. 2009. DOI: http:// dx.doi.org/10.1016/j.jallcom.2009.07.025.

[6] K. Maaz, S. Karim, A. Mumtaz et al., "Synthesis and magnetic characterization of nickel ferrite nanoparticles prepared by co-precipitation route", Journal of Magnetism and Magnetic Materials, vol. 321 (12), pp. 1838-1842, Jun. 2009. DOI: http://dx.doi.org/10.1016/j. jmmm.2008.11.098.

[7] W. Yan, W. Jiang, Q. Zhang, Y. Li and H. Wang, "Structure and magnetic properties of nickel-zinc ferrite microspheres synthesized by solvothermal method", Materials Science and Engineering B, vol. 171 (1-3), pp. 144-148, Jul. 2010. DOI: http://dx.doi.org/10.1016/j. mseb.2010.03.088.

[8] V. Figueroa-Espí, A. Alvarez-Paneque, M. Torrens, A. Otero-González and E. Reguera, "Conjugation of manganese ferrite nanoparticles to an anti Sticholysin monoclonal antibody and conjugate applications", Colloids and Surfaces A: Physicochemical and Engineering
Aspects, vol. 387 (1-3), pp. 118-124, Aug. 2011. DOI: http://dx.doi.org/10.1016/j. colsurfa.2011.08.008.

[9] E. Múzquiz-Ramos, D. A. Cortés-Hernández, O. Herrera-Romero and J. C. EscobedoBocardo, "Preparation and properties of $\mathrm{CoFe} 2 \mathrm{O} 4$ synthesized by the modified citrategel method", Materials Science Forum, vol. 644, pp. 39-42, Mar. 2010. DOI: http://dx.doi. org/10.4028/www.scientific.net/MSF.644.39.

[10] T. Kokubo and H. Takadama, "How useful is SBF in predicting in vivo bone bioactivity?", Biomaterials, vol. 27 (15), pp. 2907-2915, May. 2006. DOI: http://dx.doi.org/10.1016/j. biomaterials.2006.01.017.

[11] I. Sharifi, H. Shokrollahi and S. Amiri, "Ferritebased magnetic nanofluids used in hyperthermia applications", Journal of Magnetism and Magnetic Materials, vol. 324 (6), pp. 903-915, Mar. 2012. DOI: http://dx.doi.org/10.1016/j. jmmm.2011.10.017.

[12] E. M. Múzquiz-Ramos, D. A. Cortés-Hernández and J. C. Escobedo Bocardo, "Biomimetic apatite coating on magnetite particles", Materials Letters, vol. 64 (9), pp. 1117-1119, May. 2010. DOI: http://dx.doi.org/10.1016/j. matlet.2010.02.025.

[13] R. L. Avitia, A. Vera and L. Leija. "Estudios de la elevación de temperatura inducida por ferrofluidos en sustitutos de tejido biológico", XII Seminario Anual de Automática, Electrónica Industrial e Instrumentación, Universidad de Oviedo, pp. 515-519, 2006. 